\title{
Single-suture craniosynostosis and the epigenome: current evidence and a review of epigenetic principles
}

\author{
Othman Bin Alamer, MBBS, ${ }^{1}$ Adrian E. Jimenez, BS, ${ }^{2}$ and Tej D. Azad, MD, MS² \\ ${ }^{1}$ School of Medicine, King Saud bin Abdulaziz University for Health Sciences, Riyadh, Saudi Arabia; and 2Department of \\ Neurosurgery, Johns Hopkins Hospital, Baltimore, Maryland
}

\begin{abstract}
Craniosynostosis (CS) is a congenital disease that arises due to premature ossification of single or multiple sutures, which results in skull deformities. The surgical management of single-suture CS continues to evolve and is driven by a robust body of clinical research; however, the molecular underpinnings of CS remain poorly understood. Despite longstanding hypotheses regarding the interaction of genetic predisposition and environmental factors, formal investigation of the epigenetic underpinnings of CS has been limited. In an effort to catalyze further investigation into the epigenetic basis of CS, the authors review the fundamentals of epigenetics, discuss recent studies that shed light on this emerging field, and offer hypotheses regarding the role of epigenetic mechanisms in the development of single-suture CS.

https://thejns.org/doi/abs/10.3171/2021.1.FOCUS201008
\end{abstract}

KEYWORDS pediatric neurosurgery; craniosynostosis; epigenome; epigenetics

A MONG the many types of craniofacial anomalies, craniosynostosis (CS) is a common congenital deformity that affects newborn skulls with an incidence of approximately 1:2000. ${ }^{1}$ Due to premature calvarial suture ossification, craniosynostosis restricts skull expansion and brain growth and may lead to a variety of mental and cosmetic dysfunctions if left untreated. ${ }^{2}$ CS can result in different deformational patterns, categorized based on the suture (e.g., sagittal, coronal) and the number of sutures involved (i.e., single-suture craniosynostosis or multiple-suture craniosynostosis). ${ }^{1} \mathrm{CS}$ can also be classified based on the possible association with other malformations: syndromic CS (25\%) and nonsyndromic CS $(75 \%){ }^{3}$ While previous research has studied the clinical characteristics, management strategies, and factors associated with CS, few articles have investigated the molecular mechanisms and epigenetic basis of CS. ${ }^{4,5}$

Progress in molecular and genetic testing in the past decades has contributed to an improved understanding regarding the genetic basis of CS. ${ }^{6}$ Within the literature, more than 180 syndromes and more than 57 gene alterations have been associated with CS formation. ${ }^{7,8}$ However, the specific impact of these syndromes and gene alterations on cellular growth and intracellular signaling pathways remains unclear. ${ }^{9}$ Additionally, several articles have suggested an epigenetic basis for CS with environmental risk factors, such as smoking, advanced paternal age, and pregnancy-related complications..$^{10}$ The results of several twin studies have also emphasized the involvement of epigenetics in CS formation. ${ }^{11-13}$ While research into the epigenetic basis of CS has been undertaken, much about the specific involvement of epigenetics in CS formation remains unknown, especially regarding single-suture craniosynostosis. ${ }^{9}$

To our knowledge, a review of the current literature describing the role of epigenetics in single-suture craniosynostosis has not previously been conducted; therefore, in this article we aim to review recent investigations that have shed light on epigenetics and its mechanistic roles in single-suture synostosis development, as well as review the different genetic bases attributed to different suture synostoses. We also review the key epigenetic principles involved in genetic regulations and cellular processes.

\section{Methods}

A systematic literature search was conducted using the MEDLINE/PubMed electronic database and the references of relevant articles from inception until November 1, 2020 (Fig. 1). To identify relevant articles that address epigenetics in CS, the following search phrase was applied: "(epigenome OR epigenetics OR DNA methylation OR histone modification) AND (craniosynostosis OR suture synostosis)." Preestablished exclusion and inclusion

ABBREVIATIONS CS = craniosynostosis; DNMT = DNA methyltransferase; EZH2 = enhancer of zeste homolog 2; MZ = monozygotic; PCR = polymerase chain reaction; qPCR = quantitative PCR; siRNA = small interfering RNA. 


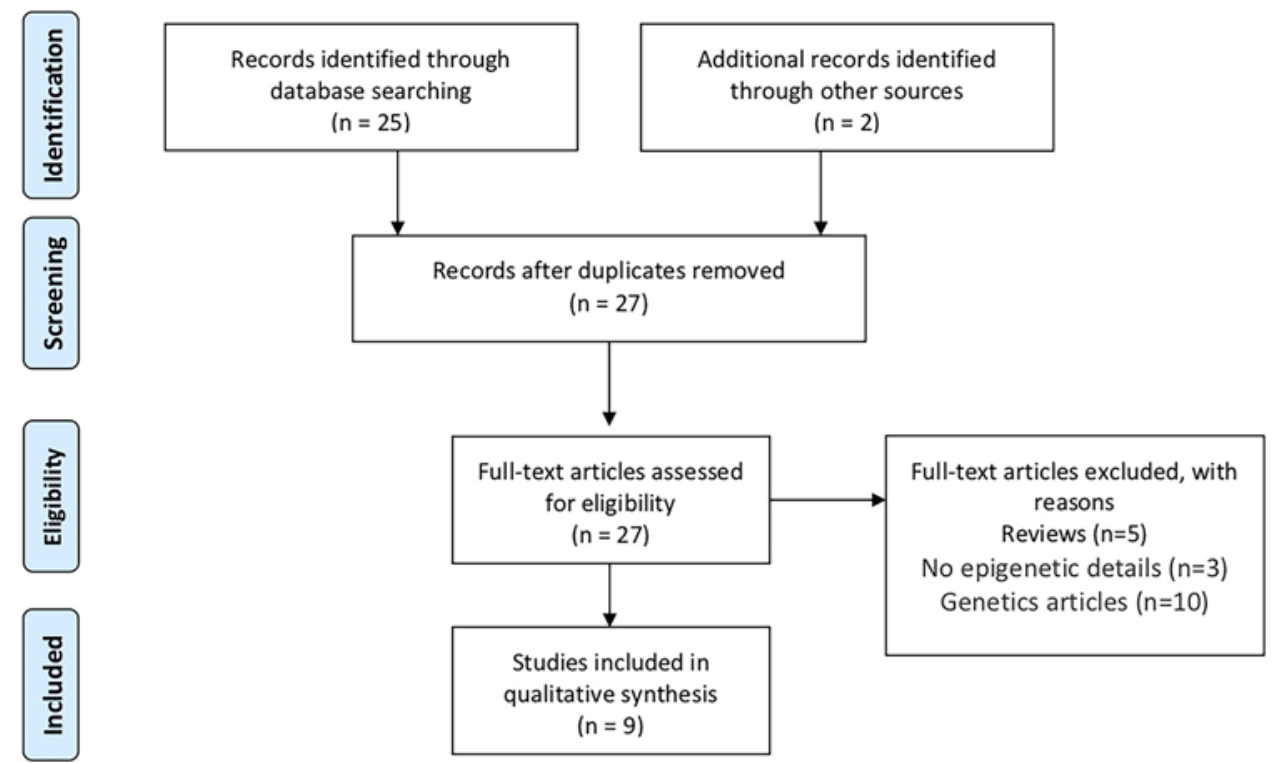

FIG. 1. PRISMA flowchart illustrating the literature review strategy. Excluded papers were narrative reviews $(n=5)$, pure genetics articles $(n=10)$, or those that did not contain epigenetics data $(n=1)$. Systematic reviews were retained $(n=1)$.

criteria were defined. Articles were included if they 1) investigated epigenetics or identified an association between epigenetics and CS; and 2) were published in English. Articles investigating the role of epigenetics in CS were retained. Articles were excluded if they 1 ) were narrative reviews or editorials; 2 ) were purely genetics articles without discussion of CS epigenetics; or 3) did not identify sufficient data.

\section{Results \\ Genetics of CS}

Illuminating the underlying causes of premature suture ossification remains a key area of study, with ongoing efforts aimed at investigating the genetic and molecular determinants of CS. Research has documented over 55 genetic alterations that have been associated with CS. ${ }^{14}$ This review focuses specifically on epigenetic alterations associated with nonsyndromic single-suture CS; however, it is important to note that different articles have reviewed the genetic bases and their implications in CS extensively. ${ }^{9}$ Nonsyndromic CS represents $75 \%$ of all craniosynostosis cases, ${ }^{15}$ with $20 \%$ attributed to chromosomal imbalances. ${ }^{16}$ Of all nonsyndromic CS cases, the most commonly involved sutures are sagittal, metopic, coronal, and lambdoid, respectively. ${ }^{1}$ The main genetic bases that underly nonsyndromic single-suture synostosis are summarized in Table $1.3,17-20$

\section{Fundamentals of Epigenetics}

DNA is wrapped around the primary structural unit of the human genome-the nucleosome. Each nucleosome is a building brick of chromatin, and it contains two copies of four histone proteins ( $\mathrm{H} 2 \mathrm{a}, \mathrm{H} 2 \mathrm{~b}, \mathrm{H} 3$, and $\mathrm{H} 4)$ and a 146-base-pair DNA. ${ }^{21}$ Two chromatin forms have been identified, euchromatin (transcriptionally active) and heterochromatin (transcriptionally inactive). ${ }^{21}$ Modifications to DNA (e.g., methylation) and histones (e.g., acetylation) alter chromatin configuration and, consequently, transcriptional activity. ${ }^{22}$ Therefore, these modifications, in addition to noncoding RNAs, control gene expression activity without altering the DNA sequence, representing a new area of study: epigenetics. ${ }^{36}$ It was not until 1942 that the word "epigenetics" was introduced to the literature by Waddington. ${ }^{24}$ Since then, the term has evolved into the definition we know today: "The study of various intracellular factors that have an effect on the stability of developmental processes through their action on genome potentialities." ${ }^{23}$

\section{DNA Methylation}

One of the best characterized epigenetic mechanisms is DNA methylation, a chemical reaction in which DNA methyltransferases (DNMTs) add a methyl group to a cysteine nucleotide that is usually followed by a guanine $(\mathrm{CpG}){ }^{43}$ While most of $\mathrm{CpG}$ dinucleotides are methylated and scattered throughout the genome, $30 \%$ are unmethylated and present in clusters known as $\mathrm{CpG}$ islands, which are found predominantly upstream from DNA transcriptional sites (i.e., at promoter or enhancer regions). ${ }^{25}$ Methylation of those $\mathrm{CpG}$ islands can, thereby, control gene expression and cellular growth. ${ }^{26}$ Thus far, literature has identified different types of DMNT, including DNMT1, DMNTa3, and DMNTb3, and characterized their functions. ${ }^{27}$ These enzymes are critical in cellular function and are involved in several disease processes. ${ }^{25} \mathrm{In}$ a recent study, Ley et al. reported an increased rate of DNMT3A mutations in patients with acute myeloid leukemia. ${ }^{28}$

\section{Histone Modifications}

Histones function to arrange DNA within the nucleus 
TABLE 1. Overview of the main genes underlying nonsyndromic single-suture synostosis formation

\begin{tabular}{|c|c|c|}
\hline Gene & Function \& Summary of Previous Reports & Sutures Involved \\
\hline SMAD6 & $\begin{array}{l}\text { SMAD6 protein is involved in intracellular transduction, gene transcription, \& signaling pathway regulation. It also regulates } \\
\text { BMP \& TGF- } \beta \text { /activin-signaling pathways, \& mutations have been implicated in syndromic \& nonsyndromic sagittal CS. }{ }^{17}\end{array}$ & Sagittal, metopic \\
\hline IL11RA & IL11RA is a constituent of the interleukin 11 receptor \& involved in extracellular signal transduction..$^{18}$ & Sagittal, metopic \\
\hline TCF12 & TCF12 is found in various intracellular signaling processes and acts as an SMAD cofactor \& heterodimerizes w/ TWIST. ${ }^{3}$ & Coronal \\
\hline ZIC1 & $\begin{array}{l}\text { ZIC1 encodes for } 1 \text { member of the zinc family that functions as a transcriptional activator \& plays a significant role in } \\
\text { CNS \& craniofacial development. }{ }^{19}\end{array}$ & Coronal \\
\hline EFNA4 & $\begin{array}{l}\text { EFNA4 is attached to the cell membrane by glycosylphosphatidylinositol. EFNA4 protein is a multidomain protein that } \\
\text { encodes \& interacts w/ tyrosine kinase receptors. }{ }^{20}\end{array}$ & Coronal \\
\hline
\end{tabular}

$\mathrm{BMP}=$ bone morphogenetic protein; TGF = transforming growth factor .

and are critical constituents of nucleosomes. ${ }^{29}$ By modifying the N-terminal tails of histone proteins, histone modification is another method to influence gene transcriptions. ${ }^{30}$ Of all histone modifications that have been characterized, only four have been well understood: methylation, acetylation, ubiquitination, and phosphorylation. ${ }^{29}$ Each type of modification is catalyzed by different enzymes and leads to different cellular consequences. Various diseases have been associated with defected histone modification enzymes, and different clinical applications relevant to these enzymes have been reported. For example, disturbances to the normal methylation of multiple histones (H3K4me1, H3K4me2, H3K4me3, and H3K9me1) have been associated with several T-cell malignancies. ${ }^{31}$ Recent studies reported promising evidence that histone acetyltransferase inhibitors and activators, as well as histone deacetylase activators, can advance breast cancer therapy and improve patient outcomes. ${ }^{32}$

\section{Noncoding RNAs}

Additionally, noncoding RNAs, which are another im- portant element of epigenetic regulation, control expression at the gene and chromosomal levels and include four main types: small interfering RNA (siRNA), microRNA (miRNA), piwi-interacting RNA (piRNA), and long noncoding RNA (lncRNA). ${ }^{33}$ Although all types are involved in epigenetic machinery, each one functions differently. miRNA is a single-strand RNA that mainly regulates gene expression at the posttranscriptional level and restricts histone-modifying enzymes. ${ }^{34}$ Unlike miRNA, which is expressed in mature mammalian cells, piRNAs regulate stem cell and embryonic tissue differentiation. ${ }^{35}$ Clinically, noncoding RNAs, especially miRNA, have been used as diagnostic and prognostic biomarkers for diseases such as type 2 diabetes and CNS injuries where miRNA expression profiles are altered..$^{36,37}$

\section{Tools and Techniques to Study Epigenetics}

Various tools and techniques are used to integrate the epigenome and analyze protein/DNA interplay qualitatively and quantitatively. DNA methylation analysis is a common technique that studies epigenetics using sodium

TABLE 2. Overview of the main studies investigating the role of epigenetics in CS formation

\begin{tabular}{|c|c|c|}
\hline Authors \& Year & Study Type & Summary \\
\hline Farooq et al., $2020^{11}$ & Case series & $\begin{array}{l}4 \text { sets of twins were investigated w/ CS; } 2 \text { twin sets were MZ \& developed different phenotypes w/ } \\
\text { variable severity. }\end{array}$ \\
\hline Magge et al., $2017^{12}$ & Case report & An MZ twin pair w/ a discordant presentation; 1 newborn w/ metopic CS. \\
\hline Lakin et al., $2012^{13}$ & Systematic review & $\begin{array}{l}\text { This article showed that different findings support the involvement of epigenetics in CS: } 62 \% \text { of twin } \\
\text { pairs in the study presented w/ discordant phenotypic features such as different sutures involved } \\
\text { \& variable severity; 2-fold increased risk in males; as phenotypic variability among MZ twins. }\end{array}$ \\
\hline Gill et al., $2012^{54}$ & Case-control & The authors report a 2-fold risk increase of CS w/ advanced maternal age ( $\geq 40 \mathrm{yrs}$ ). \\
\hline Sanchez-Lara et al., $2010^{57}$ & Case-control & $\begin{array}{l}\text { This article identified } 4 \text { maternal \& fetal factors related to CS, including plurality, preterm delivery, } \\
\text { nulliparity, macrosomia (birth weight }>4000 \mathrm{~g} \text { ), \& low birth weight. }\end{array}$ \\
\hline Oppenheimer et al., $2009^{58}$ & In vivo experimental & $\begin{array}{l}\text { Using animal models, the authors associated head compression w/ sagittal CS after observing } \\
\text { histological ossification in mice exposed to head compression. }\end{array}$ \\
\hline Oppenheimer et al., $2012^{48}$ & In vivo experimental & Using a similar methodology, the authors documented histological ossification in 4 of 6 subjects. \\
\hline Barreto et al., $2017^{4}$ & Basic since research & $\begin{array}{l}\text { This study indicates gene expression variations btwn fused \& patent sutures, \& proposed that } \\
\text { extracellular environment stiffening could accelerate ossifications \& lead to premature skull } \\
\text { bone fusion. }\end{array}$ \\
\hline Dudakovic et al., $2015^{5}$ & Basic since research & $\begin{array}{l}\text { Using chemical inhibition \& knockout models, the authors reported enhancement of osteogenic } \\
\text { commitment in human progenitor mesenchymal cells after inhibiting H3K27 methyltransferase } \\
(\mathrm{EZH} 2) \text { \& documented development of CSs. }\end{array}$ \\
\hline
\end{tabular}


bisulfite ${ }^{38}$ Subsequently, methylated cysteines are identified by different techniques, including bisulfite sequencing polymerase chain reaction (PCR), pyrosequencing, methylation-specific PCR (MS-PCR), MethyLight assay, microarray, mass spectrometry, and next-generation sequencing. ${ }^{38}$ While bisulfite PCR, MS-PCR, and MethyLight assay interrogate epigenetics in a gene-specific approach, other techniques such as microarray, mass spectrometry, and next-generation sequencing focus on genome-wide analysis. 39

Analyzing the interaction between proteins (e.g., histones) and DNA is an alternative approach used to investigate the epigenome. ${ }^{40}$ Chromatin immunoprecipitation combined with DNA sequencing (ChIP-Seq) uses antibodies to target specific proteins, with subsequent gene expression analysis conducted using PCR, quantitative PCR (qPCR), microarray, or sequencing. ${ }^{41}$ This method associates certain proteins and histone modifications with specific genes and chromosomal regions. ${ }^{42}$ At its core, ChIP-Seq enables researchers to determine if a protein is bound to a piece of DNA and is thus a critical technology for assessing the role of transcription factors. Another key epigenomic technology is the Assay for TransposaseAccessible Chromatin using sequencing (ATAC-Seq). ${ }^{43}$ ATAC-seq is an experiment that allows researchers to determine if DNA is in a region of open chromatin or closed chromatin.

\section{Epigenetics of CS}

Although relatively little is known about the epigenetic basis in CS, recent studies have begun to investigate this relationship (Table 2). Detailed epigenetic mechanisms have been established in several diseases such as cancer, cardiovascular disease, obesity, and diabetes..$^{29,36,44}$ Considering these findings, and in light of recent supporting evidence, several authors have posited the hypothesis of an epigenetic basis in the development of CS..$^{10,45}$ Importantly, only $20 \%$ of CS cases are attributed to genetic causes. ${ }^{9}$ Recent studies have associated DNA methylation in ovum and sperm with advanced age, partially explaining the high incidence of fetal abnormalities in late pregnancies. ${ }^{46,47}$ Additionally, a small number of experimental, animal model, and twin studies have shown some evidence of epigenetic modifications in CS pathogenesis. ${ }^{5,11,48}$

Barring a postzygotic mutation resulting in mosaicism, monozygotic (MZ) twins have virtually identical genomes. Therefore, differences in phenotypes between MZ twins suggests the involvement of epigenetic causes. ${ }^{49}$ Several studies report phenotypic discordance among MZ twins, raising questions about the interplay of genetics and environmental factors. One study found DNA methylation and histone acetylation in one-third of MZ twin pairs with discordant presentations. ${ }^{50}$ Furthermore, among the general population, an increased incidence rate of CS among twins compared to healthy twins has been recognized. ${ }^{13}$ To evaluate phenotypic concordance among twins with CS, Farooq et al. conducted a case series investigating four sets of twins with CS. Three twin pairs were MZ; two developed variable phenotypes with different levels of severity and management plans. ${ }^{11}$ Similarly, Magge et al. reported discordance in a genetically identical twin pair.
One newborn was healthy, whereas the other newborn developed metopic craniosynostosis. ${ }^{12}$ A systematic review by Lakin et al. showed that $62 \%$ of twin pairs with CS in the study presented with discordant phenotypic features such as different sutures involved or variable severity. ${ }^{13}$ While this study showed a higher concordance rate among MZ twins than dizygotic twins, confirming the genetic role of craniosynostosis, MZ twins were incompletely concordant, supporting the hypothesis of epigenetics. The study also reported high male predominance $(65.3 \%)$ and indicated that twinning was 2.62 times greater in patients with craniosynostosis than unaffected controls of the general population. Together, these results further support the hypothesis of epigenetics involvement in CS formation.

Different maternal, paternal, pregnancy-related, and environmental factors have been established to contribute to CS formation mediated by epigenetic machinery. ${ }^{10}$ Prior research has associated CS with maternal vitamin D deficiency, cigarette smoking, and teratogens such as phenytoin and fluconazole. ${ }^{45}$ While most commonly found in the setting of maternal autoimmune thyroid disease, congenital hyperthyroidism and craniosynostosis have been reported frequently in the literature; however, a cause-andeffect relationship has not been established. ${ }^{51,52}$ Moreover, the effect of maternal age on cranial suture synostosis has been controversial. While Loane et al. did not find an association, Gill et al. reported a twofold risk increase of craniosynostosis with advanced maternal age ( $\geq 40$ years), consistent with US national data. ${ }^{53,54}$ Similarly, advanced paternal age has also been linked with CS development. ${ }^{55}$ Although a definitive consensus has not been established, convincing evidence demonstrates that fetal constraint is a significant CS risk factor. ${ }^{56}$ In a multisite case-control study of congenital defects with a large sample size (case group, $n=675$; control group, $n=5958$ ), Sanchez-Lara et al. used the data from the National Birth Defects Prevention Study and assessed the association of CS with four factors related to fetal constraint: plurality (twins or higher), macrosomia (birth weight $>4000 \mathrm{~g}$ ), postterm gestational age ( $>42$ weeks), and nulliparity. ${ }^{57}$ Suture-specific analyses showed that plurality and nulliparity were associated with a twofold increased risk of metopic craniosynostosis, and macrosomia was associated with twice the risk of coronal craniosynostosis. However, the authors did not find an association between CS and postterm gestational age. It is worth mentioning that low birth weight was associated with metopic craniosynostosis, and preterm delivery was associated with sagittal and metopic craniosynostosis, in contrast to the authors' hypothesis.

Investigating the impact of head compression in a preclinical model, Oppenheimer et al. conducted experiments to determine if force loading of the murine calvaria would drive suture fusion in cocultured calvarial specimens, supporting the role of paracrine signaling in $\mathrm{CS}^{48,58}$ Forceloading appliances were applied to harvest calvarial coupons from postnatal mice. The experimental group underwent controlled cyclical load, applied perpendicular on the sagittal sutures. Unlike the control "no load" group, the experimental group showed histological evidence of premature suture synostosis. Moreover, cocultured calvarial coupons were more likely to exhibit histological signs for 
suture synostosis than specimens cocultured with the "no load" control group. Taken together, these results suggest that fetal constraint and biomechanical forces may be involved in the genesis of CS.

Although many authors have investigated the association between different epigenetic factors and CS development, few studies have looked into the molecular and mechanistic details. ${ }^{56,59}$ Barreto et al. ${ }^{4}$ investigated the effect of altered microenvironmental stiffness in the osteogenic commitment of suture cells and compared the genetic expression of cells from patent and fused sutures of CS. Using PCR array, variable mechanoresponses among those cells were further investigated and indicated gene upregulations caused by microenvironmental stiffness. The authors indicated an augmented expression of IGFI and TSHZ2 genes in cells from fused sutures, and given their role in bone formation, the upregulation of these genes suggests their important role in accelerated ossification and possible involvement in CS formation. In addition to IGF1 and TSHZ2, this study also reported stiffness-induced upregulation of WIF1, NOX1, BMP6,IL1 $\beta$, and $M M P 9$ expression-WIF1, NOX1, and BMP6 control osteogenic differentiation; ILI $\beta$ mediates activating inflammation; $M M P 9$ is involved in the breakdown of extracellular matrix - using qPCR analysis. Furthermore, the authors observed a notable increase of expression in these genes when cells from fused sutures were cultured with an osteogenic medium. Highlighting the role of altered force transmission in defining the timing and magnitude of suture synostosis, this study opened opportunities to explore further the potential mechanotransductive mechanisms associated with CS.

Dudakovic et al. ${ }^{5}$ examined the expression of a large cohort of epigenetic regulators $(>300)$ during osteogenic differentiation of human mesenchymal cells to determine critical epigenetic regulators involved in osteogenic commitment. Enhancer of zeste homolog 2 (EZH2) is a methyltransferase enzyme that catalyzes trimethylation of histone 3 lysine 27 (H3K27me3), and despite its pivotal role during normal skeletal development, genetic analyses indicated downregulation of this enzyme during osteogenic differentiation. Testing the hypothesis, Dudakovic et al. used a small molecule inhibitor and siRNA knockdown to evaluate the consequence of EZH2 inhibition on osteogenic differentiation. As hypothesized, blocking EZH2 in vivo stimulated osteogenic differentiation and suppressed adipogenic differentiation, suggesting that EZH2 is a crucial regulator of osteogenic commitment and skeletal development. However, in addition to identifying other epigenetic regulators, further illuminating the enzyme mechanism is warranted to better understand the process and subsequently proceed to clinical applications. The results of the studies mentioned above are of significant importance because epigenetic targets are used to treat multiple types of cancer as well as various other medical conditions including mood disorders and epilepsy, autoimmune diseases, pain, and Alzheimer's disease. ${ }^{60-63}$ Targeting some of these enzymes and proteins (e.g., EZH2) can be leveraged to inhibit ossification and, therefore, interfere with suture-synostosis pathogeneses, opening up avenues to improve outcomes and prognosis in patients with CS.

\section{Conclusions}

As with many diseases, a comprehensive understanding of the fundamental epigenetic mechanisms underlying CS development can aid in disease prevention and treatment, and therefore improve patient outcomes. In this article, we have reviewed the current scientific literature investigating the role of epigenetics in CS. Thus far, little has been established about the epigenetics involved in CS and its interplay with genetic and environmental factors. Therefore, further research is necessary to reveal the critical molecular aspects of epigenetic regulation underlying CS development.

\section{References}

1. Johnson D, Wilkie AOM. Craniosynostosis. Eur J Hum Genet. 2011;19(4):369-376.

2. Alden TD, Lin KY, Jane JA. Mechanisms of premature closure of cranial sutures. Childs Nerv Syst. 1999;15(11-12):670-675.

3. Lattanzi W, Barba M, Di Pietro L, Boyadjiev SA. Genetic advances in craniosynostosis. Am J Med Genet A. 2017;173(5): 1406-1429.

4. Barreto S, González-Vázquez A, R Cameron A, et al. Identification of stiffness-induced signaling mechanisms in cells from patent and fused sutures associated with craniosynostosis. Sci Rep. 2017;7(1):11494.

5. Dudakovic A, Camilleri ET, Xu F, et al. Epigenetic control of skeletal development by the histone methyltransferase Ezh2. J Biol Chem. 2015;290(46):27604-27617.

6. Miller KA, Twigg SRF, McGowan SJ, et al. Diagnostic value of exome and whole genome sequencing in craniosynostosis. J Med Genet. 2017;54(4):260-268.

7. Kimonis V, Gold JA, Hoffman TL, et al. Genetics of craniosynostosis. Semin Pediatr Neurol. 2007;14(3):150-161.

8. Twigg SRF, Wilkie AOM. A genetic-pathophysiological framework for craniosynostosis. Am J Hum Genet. 2015; 97(3):359-377.

9. Armand T, Schaefer E, Di Rocco F, et al. Genetic bases of craniosynostoses: an update. Neurochirurgie. 2019;65(5):196-201.

10. Feil R, Fraga MF. Epigenetics and the environment: emerging patterns and implications. Nat Rev Genet. 2012;13(2):97-109.

11. Farooq S, Morton J, Lloyd M, Krishna ST. The influence of epigenetic factors in four pairs of twins with non-syndromic craniosynostosis. J Craniofac Surg. 2020;31(1):283-285.

12. Magge SN, Snyder K, Sajja A, et al. Identical twins discordant for metopic craniosynostosis: evidence of epigenetic influences. J Craniofac Surg. 2017;28(1):14-16.

13. Lakin GE, Sinkin JC, Chen R, et al. Genetic and epigenetic influences of twins on the pathogenesis of craniosynostosis: a meta-analysis. Plast Reconstr Surg. 2012;129(4):945-954.

14. Al-Rekabi Z, Cunningham ML, Sniadecki NJ. Cell mechanics of craniosynostosis. ACS Biomater Sci Eng. 2017;3(11): 2733-2743.

15. Greenwood J, Flodman P, Osann K, et al. Familial incidence and associated symptoms in a population of individuals with nonsyndromic craniosynostosis. Genet Med. 2014;16(4): 302-310.

16. Passos-Bueno MR, Fanganiello RD, Jehee FS. Craniosynostosis and chromosomal alterations. Monogr Hum Genet. 2011;19:152-164.

17. Calpena E, Cuellar A, Bala K, et al. SMAD6 variants in craniosynostosis: genotype and phenotype evaluation. Genet Med. 2020;22(9):1498-1506.

18. Keupp K, Li Y, Vargel I, et al. Mutations in the interleukin receptor ILI1RA cause autosomal recessive Crouzon-like craniosynostosis. Mol Genet Genomic Med. 2013;1(4):223237. 
19. Gaston-Massuet C, Henderson DJ, Greene NDE, Copp AJ. Zic4, a zinc-finger transcription factor, is expressed in the developing mouse nervous system. Dev Dyn. 2005;233(3): $1110-1115$.

20. Lee E, Le T, Zhu Y, et al. A craniosynostosis massively parallel sequencing panel study in 309 Australian and New Zealand patients: findings and recommendations. Genet Med. 2018;20(9):1061-1068.

21. Luger K, Mäder AW, Richmond RK, et al. Crystal structure of the nucleosome core particle at $2.8 \AA$ resolution. Nature. 1997;389(6648):251-260.

22. Huisinga KL, Brower-Toland B, Elgin SCR. The contradictory definitions of heterochromatin: transcription and silencing. Chromosoma. 2006;115(2):110-122.

23. Nicoglou A, Merlin F. Epigenetics: a way to bridge the gap between biological fields. Stud Hist Philos Biol Biomed Sci. 2017;66:73-82.

24. Waddington CH. The epigenotype. 1942. Int J Epidemiol. 2012;41(1):10-13.

25. Robertson KD. DNA methylation and human disease. Nat Rev Genet. 2005;6(8):597-610.

26. Berger SL. The complex language of chromatin regulation during transcription. Nature. 2007;447(7143):407-412.

27. Feng J, Zhou Y, Campbell SL, et al. Dnmt1 and Dnmt3a maintain DNA methylation and regulate synaptic function in adult forebrain neurons. Nat Neurosci. 2010;13(4):423-430.

28. Ley TJ, Ding L, Walter MJ, et al. DNMT3A mutations in acute myeloid leukemia. $N$ Engl J Med. 2010;363(25):24242433.

29. Dawson MA, Kouzarides T. Cancer epigenetics: from mechanism to therapy. Cell. 2012;150(1):12-27.

30. Murray K. The occurrence of $\varepsilon-\mathrm{N}$-methyl lysine in histones. Biochemistry. 1964;3(1):10-15.

31. Barski A, Cuddapah S, Cui K, et al. High-resolution profiling of histone methylations in the human genome. Cell. 2007; 129(4):823-837.

32. Guo P, Chen W, Li H, et al. The histone acetylation modifications of breast cancer and their therapeutic implications. Pathol Oncol Res. 2018;24(4):807-813.

33. Morris KV, Chan SWL, Jacobsen SE, Looney DJ. Small interfering RNA-induced transcriptional gene silencing in human cells. Science. 2004;305(5688):1289-1292.

34. Ruvkun G. Molecular biology. Glimpses of a tiny RNA world. Science. 2001;294(5543):797-799.

35. Lau NC, Seto AG, Kim J, et al. Characterization of the piRNA complex from rat testes. Science. 2006;313(5785): 363-367.

36. Ling C, Rönn T. Epigenetics in human obesity and type 2 diabetes. Cell Metab. 2019;29(5):1028-1044.

37. Sun P, Liu DZ, Jickling GC, et al. MicroRNA-based therapeutics in central nervous system injuries. J Cereb Blood Flow Metab. 2018;38(7):1125-1148.

38. Mulero-Navarro S, Esteller M. Epigenetic biomarkers for human cancer: the time is now. Crit Rev Oncol Hematol. 2008; 68(1):1-11.

39. Laird PW. Principles and challenges of genomewide DNA methylation analysis. Nat Rev Genet. 2010;11(3):191-203.

40. Orlando V. Mapping chromosomal proteins in vivo by formaldehyde-crosslinked-chromatin immunoprecipitation. Trends Biochem Sci. 2000;25(3):99-104.

41. Goto Y, Gomez M, Brockdorff N, Feil R. Differential patterns of histone methylation and acetylation distinguish active and repressed alleles at X-linked genes. Cytogenet $G e$ nome Res. 2002;99(1-4):66-74.

42. Kim YH, Marhon SA, Zhang Y, et al. Rev-erb $\alpha$ dynamically modulates chromatin looping to control circadian gene transcription. Science. 2018;359(6381):1274-1277.

43. Buenrostro JD, Giresi PG, Zaba LC, et al. Transposition of native chromatin for fast and sensitive epigenomic profiling of open chromatin, DNA-binding proteins and nucleosome position. Nat Methods. 2013;10(12):1213-1218.

44. Ordovás JM, Smith CE. Epigenetics and cardiovascular disease. Nat Rev Cardiol. 2010;7(9):510-519.

45. Yilmaz E, Mihci E, Nur B, et al. Recent advances in craniosynostosis. Pediatr Neurol. 2019;99:7-15.

46. Milekic MH, Xin Y, O'Donnell A, et al. Age-related sperm DNA methylation changes are transmitted to offspring and associated with abnormal behavior and dysregulated gene expression. Mol Psychiatry. 2015;20(8):995-1001.

47. Markunas CA, Wilcox AJ, Xu Z, et al. Maternal age at delivery is associated with an epigenetic signature in both newborns and adults. PLoS One. 2016;11(7):e0156361.

48. Oppenheimer AJ, Rhee ST, Goldstein SA, Buchman SR. Force-induced craniosynostosis via paracrine signaling in the murine sagittal suture. J Craniofac Surg. 2012;23(2):573-577.

49. Zwijnenburg PJG, Meijers-Heijboer H, Boomsma DI. Identical but not the same: the value of discordant monozygotic twins in genetic research. Am J Med Genet B Neuropsychiatr Genet. 2010;153B(6):1134-1149.

50. Fraga MF, Ballestar E, Paz MF, et al. Epigenetic differences arise during the lifetime of monozygotic twins. Proc Natl Acad Sci U S A. 2005;102(30):10604-10609.

51. Higashino T, Hirabayashi S. A secondary craniosynostosis associated with juvenile hyperthyroidism. J Plast Reconstr Aesthet Surg. 2013;66(10):e284-e286.

52. Chawla R, Alden TD, Bizhanova A, et al. Squamosal suture craniosynostosis due to hyperthyroidism caused by an activating thyrotropin receptor mutation (T632I). Thyroid. 2015; 25(10):1167-1172.

53. Loane M, Dolk H, Morris JK. Maternal age-specific risk of non-chromosomal anomalies. BJOG. 2009;116(8):1111-1119.

54. Gill SK, Broussard C, Devine O, et al. Association between maternal age and birth defects of unknown etiology: United States, 1997-2007. Birth Defects Res A Clin Mol Teratol. 2012;94(12):1010-1018.

55. Goriely A, Wilkie AOM. Paternal age effect mutations and selfish spermatogonial selection: causes and consequences for human disease. Am J Hum Genet. 2012;90(2):175-200.

56. Bradley JP, Shahinian H, Levine JP, et al. Growth restriction of cranial sutures in the fetal lamb causes deformational changes, not craniosynostosis. Plast Reconstr Surg. 2000; 105(7):2416-2423.

57. Sanchez-Lara PA, Carmichael SL, Graham JM Jr, et al. Fetal constraint as a potential risk factor for craniosynostosis. Am J Med Genet A. 2010;152A(2):394-400.

58. Oppenheimer AJ, Rhee ST, Goldstein SA, Buchman SR. Force-induced craniosynostosis in the murine sagittal suture. Plast Reconstr Surg. 2009;124(6):1840-1848.

59. Poot M. Structural genome variations related to craniosynostosis. Mol Syndromol. 2019;10(1-2):24-39.

60. Khan O, La Thangue NB. HDAC inhibitors in cancer biology: emerging mechanisms and clinical applications. Immunol Cell Biol. 2012;90(1):85-94.

61. Chuang DM. The antiapoptotic actions of mood stabilizers: molecular mechanisms and therapeutic potentials. Ann NY Acad Sci. 2005;1053:195-204.

62. Chiechio S, Zammataro M, Morales ME, et al. Epigenetic modulation of mGlu2 receptors by histone deacetylase inhibitors in the treatment of inflammatory pain. Mol Pharmacol. 2009;75(5):1014-1020.

63. Hewagama A, Richardson B. The genetics and epigenetics of autoimmune diseases. J Autoimmun. 2009;33(1):3-11.

\section{Disclosures}

The authors report no conflict of interest concerning the materials or methods used in this study or the findings specified in this paper. 


\section{Author Contributions}

Conception and design: Azad. Acquisition of data: Bin Alamer. Drafting the article: Bin Alamer, Jimenez. Critically revising the article: all authors. Reviewed submitted version of manuscript: all authors. Approved the final version of the manuscript on behalf of all authors: Azad. Statistical analysis: Jimenez.

\section{Correspondence}

Tej D. Azad: Johns Hopkins University School of Medicine, Baltimore,MD.tazad1@jhmi.edu. 